\title{
The Mouse-Specific Gly-to-Cys Mutation in Mammalian Acrosin is a Cause of Impairment in Proteolytic Activity
}

\author{
S. Nishio and T. Matsuda
}

Background: Among eutherian mammals, only mouse has an additional cysteine residue (Cys162) next to the conserved Cys163 of acrosin, and the mouse acrosin shows exceptionally low proteolysis. Acr-nul male mice are fertile and yield litters in size comparable to wild-type ones, whereas acr-nul rats yield litters much smaller in size and show in vivo sperm penetration of egg $\mathrm{ZP}$ at a frequency much lower than the wild type. No recombinant acrosin has been obtained so far in the mature and active form.

Main Questions: Why does mouse acrosin show exceptionally low proteolytic activity? Could such low activity of mouse acrosin be ascribed to the additional Cys 162 residue?

Experimental Design: The Cys-to-Gly and Gly-to-Cys mutants as well as wild types of mouse and rat acrosin were prepared using pMT/Bip-V5-His insect cell expression system. Proteolytic activity was evaluated using gelatin-zymography, while amidase activity was determined using peptide-MCA fluorometric substrates.

Main Results: Like native ones, recombinant mouse and rat acrosins showed almost no and remarkable proteolytic activities, respectively. Reverse mutation of Cys162-to-Gly in mouse acrosin resulted in the recovery of proteolytic activity comparable to rat acrosin. Conversely, Gly162-to-Cys mutation of rat acrosin markedly impaired the proteolytic activity. Interestingly, all of these acrosins and mutants showed clear amidase activity equally. On the other hand, mutation of the conserved Cys163 to Gly severely impaired not only proteolytic activity but also amidase activity of the mouse Cys162-to-Gly acrosin.

Conclusions: Mouse may have evolved sperm by somehow adapting to acrosin activity impairment.

\footnotetext{
S. Nishio $(\square) \cdot$ T. Matsuda

Department of Applied Molecular Biosciences, Graduate School of Bioagricultural Sciences,

Nagoya University, Nagoya, Japan

e-mail: nishio.shunsuke@c.mbox.nagoya-u.ac.jp
} 